\title{
Dynamics of water at low temperatures and implications for biomolecules
}

\author{
P. Kumar ${ }^{1}$, G. Franzese ${ }^{2}$, S. V. Buldyrev ${ }^{3}$, and H. E. Stanley ${ }^{1}$ \\ 1 Center for Polymer Studies and Department of Physics, Boston University, \\ Boston, MA 02215 USA pradeep@buphy.bu.edu / hes@bu.edu \\ 2 Departament de Física Fonamental, Universitat de Barcelona, Diagonal 647, \\ Barcelona 08028, Spain gfranzese@ub.edu \\ 3 Department of Physics, Yeshiva University, 500 West 185th Street, New York, \\ NY 10033 USA buldyrev@yu.edu
}

The biological relevance of water is a puzzle that has attracted much scientific attention. Here we recall what is unusual about water and discuss the possible implications of the unusual properties of water also known as water anomalies in biological processes. We find the surprising results that some anomalous properties of water, including results of a recent experiments on hydrated biomolecules, are all consistent with the working hypothesis of the presence of a first order phase transition between two liquids with different densities at low temperatures and high pressures, which ends in a critical point. To elucidate the relation between dynamic and thermodynamic anomalies, we investigate the presence of this liquid-liquid critical point in several models. Using molecular dynamics simulations, we find a correlation between the dynamic transition and the locus of specific heat maxima $C_{P}^{\max }$ (also known as Widom line) emanating from the critical point. We investigate the relation between the dynamic transitions of biomolecules (lysozyme and DNA) and the dynamic and thermodynamic properties of hydration water. We find that the dynamic transition of the macromolecules, sometimes called "protein glass transition" in case of proteins, occurs at the same temperature where the dynamics of hydration water has a crossover, and also coincides with the temperature of maximum of specific heat and the maximum of the temperature derivative of the orientational order parameter. Since our simulations are consistent with the possibility that the protein glass transition results from a change in the behavior of hydration water, specifically from crossing the Widom line, we explore in more details the relation between the dynamic crossover and the Widom line in a tractable model for water. We find that the dynamic crossover can be fully explained as a consequence of the thermodynamic and structural changes occurring at the Widom line of water. We, therefore, argue that the so-called "glass transition" of hydrated 
proteins is just a consequence of the thermodynamic and structural changes of the surrounding water.

\section{Introduction}

Water is one of the most ubiquitous liquids and perhaps the only one which exists in all the three stable phases (liquids, solid and gas) at ambient conditions in nature. Although the chemical composition of water is very simple, the physical properties of water make it unique among the other substances. Water has an unusually high boiling and melting point as well as a very high liquid-gas critical temperature compared to the liquids belonging to the same isoelectronic structure as water. Water is also rich in the number of crystal stucture it forms at different temperatures and pressures. Indeed, more than thirteen different crystals of water (ices) have been discovered.

Many living beings can survive without water only a few days. This is because water participates in the majority of the biological processes [1], such as the metabolism of nutrients catalysed by enzymes. In order to be effective, the enzymes need to be suspended in a solvent to adopt their active three-dimensional structure. Another important role of water is that it allows the processes of elimination of cellular metabolic residues. It is water through which our cells can communicate and that oxygen and nutrients can be brought to our tissues. Nevertheless, there is no clear reason why water should have an essential role in biological processes.

What could make water more interesting compared to many other liquids are the properties of water, also called anomalies of water. Due to its very puzzling nature, water has been subject of intense studies for decades. One, regarding the well known anomaly of the density maximum at $4^{\circ} \mathrm{C}$, dates backs to the seventeenth century. It is for this anomaly that ice floats on water and fishes can survive in warm waters below a layer of ice at temperatures well below $0^{\circ} \mathrm{C}$.

In the following we will discuss the properties of water by, first, describing some of its anomalies, then by presenting a working hypothesis and, next, by testing it. In our discussion we will use different theoretical tools, including computer simulations. The recent dramatic increase of computational power makes it feasible to study the behaviour of water at long time scales using computer models, and their quantitative and qualitative agreement with the experiments been demonstrated by many studies over last few years.

\subsection{Water anomalies at low temperatures}

Pure water can been cooled well below the melting point without freezing. Such a water is called supercooled liquid water. The supercooled liquid is metastable and the free energy minimum state is the crystal state, i. e. it 
cannot last forever as a stable liquid phase, but, sooner or later, it will transform into ice.

In the supercooled region most of the anomalous properties of water get more enhanced. In Figure 1, we show a schematic representation of three different response functions of water, namely the specific heat $C_{P}$, the isothermal compressibility $\kappa_{T}$, and the coefficient of thermal expansion $\alpha_{P}$, as a function of temperature $T$ at atmospheric pressure. $C_{P}$ is a measure of enthalpy fluctuation $\left\langle(\Delta H)^{2}\right\rangle$, hence one would expect that it should decrease as the temperature is decreased, however for the case of water it increases sharply as the temperature is decreased. Similarly, since $\kappa_{T}$ is the measure of volume fluctuations $\left\langle(\Delta V)^{2}\right\rangle$, it should decrease upon decreasing the temperature. In the case of water, instead, it increases like $C_{P}$ and seems to diverge at low temperatures in experiments [2]. Figure 1 (c) shows the behavior of $\alpha_{P}$ and since $\alpha_{P}$ is the measure of cross fluctuations of volume and entropy $\langle\Delta V \Delta S\rangle$, it is positive for normal liquids. However, in the case of water it becomes negative at the temperature of maximum density $T_{M D}$ at constant pressures, suggesting that below $T_{M D}$ as the volume is increased the entropy decreases. Like other response functions, $\alpha_{P}$ also seems to diverge at low temperatures in experiments. Dashed curves in Figure 1 are the schematic representations of the behavior of normal liquids for a comparison. Since the experiments on bulk liquid water can not be performed below the homogeneous nucleation temperature $T_{H} \approx-38^{\circ}$, where the crystal formation is inevitable, it is not possible to test the seeming divergence of response functions at low temperatures. Later we will show, using computer simulations, that indeed the response functions do not diverge but have a maxima at low temperatures.

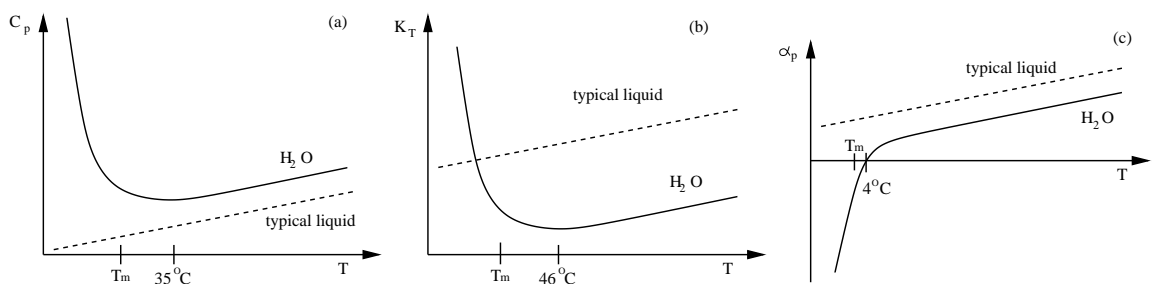

Fig. 1. Schematic representation of different response functions $C_{P}, \kappa_{T}$ and $\alpha_{P}$ of liquid water as a function of temperature $T$. The behavior of a normal liquid is shown as dashed curves.

\subsection{Interpretation of the anomalies}

Over last few decades many experiments and computer simulations of water have been performed and many hypotheses have been put forth to explain these anomalies. Some of the hypotheses tested and proved wrong and some of them are still under the scrutiny of careful experiments. There exist two 
scenarios, namely the singularity-free and liquid-liquid critical point scenario, consistent with the experiments.

In the singularity-free interpretation $[3,4]$ the large increase of response functions seen in the experiments represents only an apparent singularity, due to local density fluctuations, with no real divergence. All the anomalies are interpreted as a consequence of the negative volume-entropy cross fluctuations [4].

Another hypothesis is the presence of a phase transition, in the region of deep supercooled water, from high density liquid (HDL) water at high temperature to low density liquid (LDL) water at low temperature. The hypothesized first order transition line terminates at a critical point known as liquid-liquid critical point of water. This liquid-liquid critical point in water is estimated to be located at around pressure $P \approx 100 \mathrm{MPa}$ and temperature $T \approx 220 \mathrm{~K}[5,6]$. However this hypothesized critical point lies below the homogeneous nucleation temperature of water and hence no experiments on bulk water can be performed to locate it as water spontaneously freezes.

Although this region can not be accessed in bulk water, recent experiments on water confined in nanoscale geometries have made it possible to study liquid water in this region, since confinement suppresses the crystallization. Experiments on water confined in the pores of diameter of few nanometers, shed light on the properties of water below the homogeneous nucleation temperature $[7,8]$. Specifically it was found that on decreasing the temperature below $T \approx 225 \mathrm{~K}$, water shows a dramatic change in the behavior of the dynamics, namely a crossover in the dynamic quantities such as the diffusion coefficient or the correlation times at low $T$ below $T \approx 225 \mathrm{~K}$.

Although the origin of the crossover has different interpretations [9, 10], the experimental evidences point to indicate that the change in the dynamics is triggered by a local rearrangement of the hydrogen bond network [11].

Using molecular dynamics, in section 2 we provide evidences that this behavior in the change of dynamics of bulk water at low $T$ is consistent with the LL-critical point hypothesis. Next, in section 3 we relate this dynamic crossover for water with the "glass transition" in hydrated proteins suggesting that the changes in the protein dynamics are a consequence of the change in the behavior of the hydration water. Finally, by using a tractable water model, we show in section 5 that the dynamic crossover can be explained by structural and thermodynamic changes of water.

\subsection{Widom line}

By definition, in a first order phase transitions, thermodynamic state functions such as density $\rho$ and enthalpy $H$ discontinuously change as we cool the system along a path crossing the equilibrium coexistence line [Figure 2(a), path $\beta$ ]. In a real experiment, this discontinuous change may not occur at the coexistence line since a substance can remain in a supercooled metastable phase until a limit of stability (a spinodal) is reached [12] [Figure 2(b), path $\beta$ ]. If the system 
is cooled isobarically along a path above the liquid-gas critical pressure $P_{c}$ [Figure 2(b), path $\alpha$ ], the state functions continuously change from the values characteristic of a high temperature phase (gas) to those characteristic of a low temperature phase (liquid).

The thermodynamic response functions which are the derivatives of the state functions with respect to temperature [e.g., isobaric heat capacity $C_{P} \equiv$ $\left.(\partial H / \partial T)_{P}\right]$ have maxima at temperatures denoted by $T_{\max }(P)$. Remarkably these maxima are still prominent far above the critical pressure [17-21], and the values of the response functions at $T_{\max }(P)$ (e.g., $C_{P}^{\max }$ ) diverge as the critical point is approached. The lines of the maxima for different response functions asymptotically get closer to one another as the critical point is approached, since all response functions become expressible in terms of the correlation length. This asymptotic line is sometimes called the Widom line, and is often regarded as an extension of the coexistence line into the one-phase regime.

As we mentioned in the previous section, water's anomalies have been hypothesized to be related to the existence of a line of a first order liquid-liquid phase transition terminating at a liquid-liquid critical point $[5,6,12,22]$, located below the homogeneous nucleation line in the deep supercooled region of the phase diagram - sometimes called the "no-man's land" because it is difficult to make direct measurements on the bulk liquid phase [6]. In supercooled water, the liquid-liquid coexistence line and the Widom line have negative slopes. Thus, if the system is cooled at constant pressure $P_{0}$, computer simulations suggest that for $P_{0}<P_{c}$ [Figure 2(c), path $\alpha$ ] experimentallymeasured quantities will change dramatically but continuously in the vicinity of the Widom line (with huge fluctuations as measured by, e.g., $C_{P}$ ) from those resembling the high density liquid (HDL) to those resembling the low density liquid (LDL). For $P_{0}>P_{c}$ [Figure 2(c), path $\beta$ ], experimentally-measured quantities will change discontinuously if the coexistence line is actually seen. However, the coexistence line can be difficult to detect in a pure system due to metastability, and changes will occur only when the spinodal is approached where the HDL phase is no longer stable. The changes in behavior may include not only static quantities like response functions [17-21] but also dynamic quantities like diffusivity.

In the case of water, a significant change in dynamical properties has been suggested to take place in deeply supercooled states [2, 22-26]. Unlike other network forming materials [27], water behaves as a non-Arrhenius liquid in the experimentally accessible window [22, 28, 29]. A dynamics is Arrhenius if the relaxation time is well described by

$$
\tau=\tau_{0} \exp \left[\frac{E_{A}}{k_{B} T}\right],
$$

where $\tau_{0}$ is the relaxation time in the large- $T$ limit, $k_{B}$ is the Boltzmann constant, and $E_{\mathrm{A}}$ is the $T$-independent activation energy. If $E_{\mathrm{A}}$ depends on $T$, the dynamics is non-Arrhenius . 


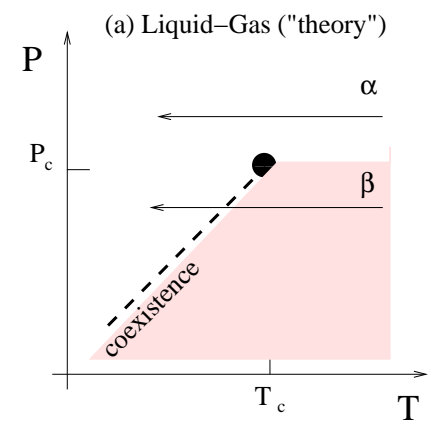

(c) Water

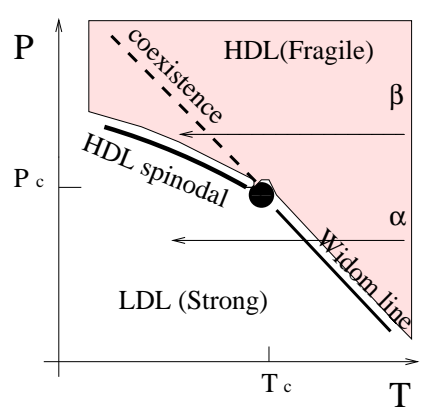

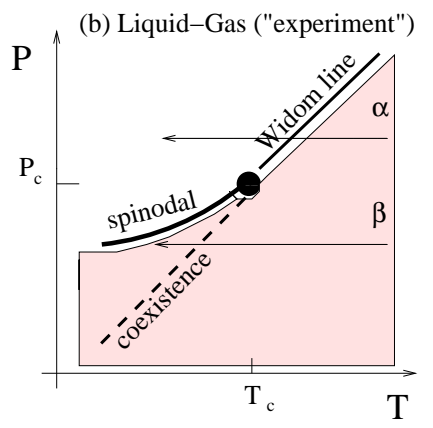

(d) Jagla Model

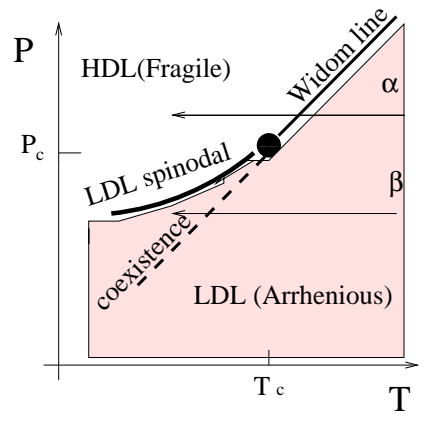

Fig. 2. (a) Schematic phase diagram for the critical region associated with a liquidgas critical point. Shown are the two features displaying mathematical singularities, the critical point (closed circles) and the liquid-gas coexistence (bold dashed curve). (b) Same as (a) with the addition of the gas-liquid spinodal and the Widom line. Along the Widom line, thermodynamic response functions have extrema in their $T$ dependence. The path $\alpha$ denotes a path along which the Widom line is crossed. Path $\beta$ denotes a path meeting the coexistence line. (c) A hypothetical phase diagram for water of possible relevance to the recent neutron scattering experiments by Chen et al. $[7,13]$ on confined water. The liquid-liquid coexistence, which has a negative sloped coexistence line, generates a Widom line which extends below the critical point, suggesting that water may exhibit a dynamic crossover (non-Arrhenius-toArrhenius) transition for $P<P_{c}$ (path $\alpha$ ), while no dynamic changes will occur above the critical point (path $\beta$ ). (d) A sketch of the $P-T$ phase diagram for the two-scale Jagla model (see text). For the Jagla potential, as well as for the doublestep potential [14] and the shouldered potential $[15,16]$, the liquid-liquid phase transition line has a positive slope. Upon cooling at constant pressure above the critical point (path $\alpha$ ), the liquid changes from a low density state (characterized by a non-glassy Arrhenius dynamics) to a high density state (characterized by glassy Arrhenius dynamics with much larger activation energy) as the path crosses the Widom line. Upon cooling at constant pressure below the critical point (path $\beta$ ), the liquid remains in the LDL phase as long as path $\beta$ does not cross the LDL spinodal line. Thus one does not expect any change in the dynamic behavior along the path $\beta$, except upon approaching to glass transition at lower $T$, where one can expect the non-Arrhenius behavior. From ref. [8] 
Based on analogies with other network forming liquids and with the thermodynamic properties of the amorphous forms of water, it has been suggested that, at ambient pressure, liquid water should show a dynamic crossover from non-Arrhenius behavior at high $T$ to Arrhenius behavior at low $T$ [24, 30-34]. Using Adam-Gibbs theory [35], the dynamic crossover in water was related to the $C_{P}^{\max }$ line $[23,36]$. Also, a dynamic crossover has been associated with the liquid-liquid phase transition in simulations of silicon and silica [37, 38]. Recently a dynamic crossover in confined water was studied experimentally $[7,13,39,40]$ since nucleation can be avoided in confined geometries. Here, we report the recent investigation of experiments on water $[7,13,40]$ as arising from the presence of the hypothesized liquid-liquid critical point, which gives rise to a Widom line and an associated dynamics crossover [Figure 2(c) and $2(\mathrm{~d})$, path $\alpha$ ].

\section{Bulk water}

Using molecular dynamics simulations [41], Xu et al. [8] studied three models, each of which has a liquid-liquid critical point. Two of the models (the TIP5P [42] and the ST2 [43]) treat water as a multiple site rigid body, interacting via electrostatic site-site interactions complemented by a Lennard-Jones potential. The third model is the spherical "two-scale" Jagla potential with attractive and repulsive ramps which has been studied in the context of liquidliquid phase transitions and liquid anomalies [21, 31-33, 44, 45]. For all three models, $\mathrm{Xu}$ et al. evaluated the loci of maxima of the relevant response functions, compressibility and specific heat, which coincide close to the critical point and give rise to the Widom line. They found evidence that, for all three potentials, the dynamic crossover occurs just when the Widom line is crossed (Figure 2).

These findings are consistent with the possibility that the observed dynamic crossover along path $\alpha$ is related to the behavior of $C_{P}$, suggesting that enthalpy or entropy fluctuations may have a strong influence on the dynamic properties [21, 23, 38]. Indeed, as the thermodynamic properties change from the high-temperature side of the Widom line to the low-temperature side, $(\partial S / \partial T)_{P}=C_{P} / T>0$ implies that the entropy must decrease. The entropy decrease is most pronounced at the Widom line when $C_{P}=C_{P}^{\max }$. Since the configurational part of the entropy, $S_{\text {conf }}$, makes the major contribution to $S$, we expect that $S_{\text {conf }}$ also decreases sharply on crossing the Widom line.

According to Adam-Gibbs theory [35], the diffusivity $D$ changes as

$$
D \sim \exp \left[-\frac{A}{T S_{\text {conf }}}\right] \text {. }
$$

Hence, we expect that $D$ sharply decreases upon cooling at the Widom line. If $S_{\text {conf }}$ does not change appreciably with $T$, then the Adam-Gibbs equation 

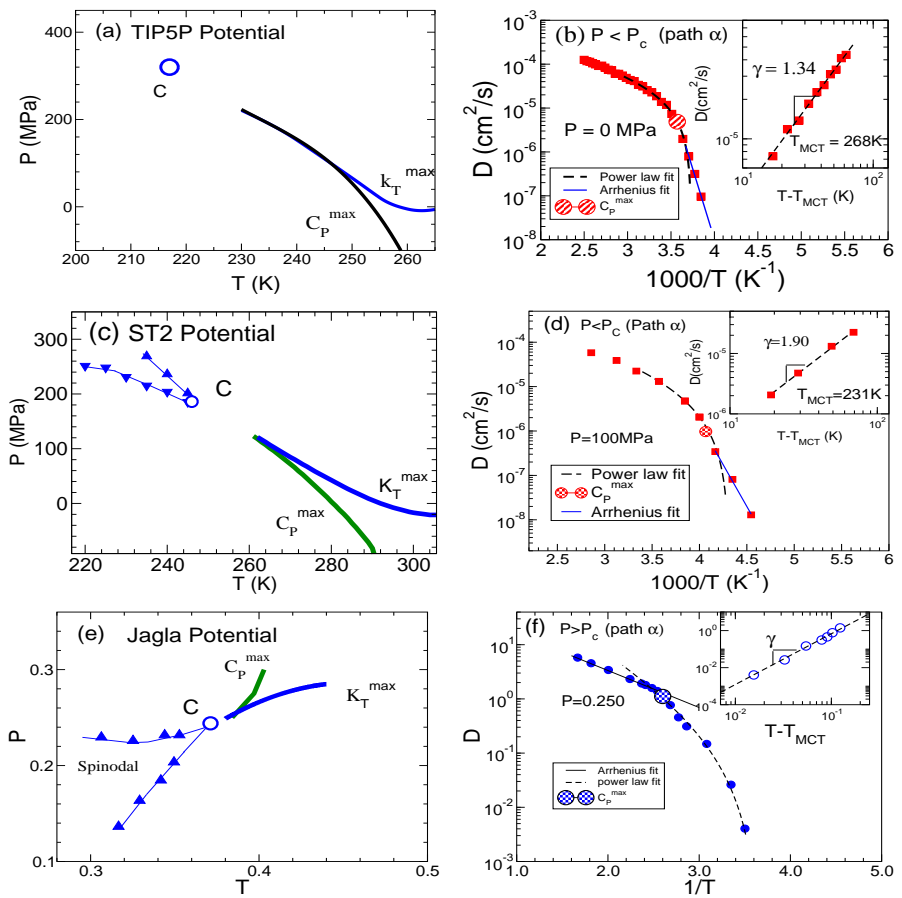

Fig. 3. (a) Relevant part of the phase diagram for the TIP5P water potential, showing the liquid-liquid critical point $C$ at $P_{c}=320 \mathrm{MPa}$ and $T_{c}=217 \mathrm{~K}$, the line of maximum of isobaric specific heat $C_{P}^{\max }$ and the line of maximum of isothermal compressibility $K_{T}^{\max }$. (b) $D$ as a function of $T$ for $P=100 \mathrm{MPa}$ (path $\alpha$ ). At high temperatures, $D$ behaves like that of a non-Arrhenius liquid and can be fit by $D \sim\left(T-T_{M C T}\right)^{\gamma}$ (also shown in the inset) where $T_{M C T}=220 \mathrm{~K}$ and $\gamma=1.942$, while at low temperatures the dynamic behavior changes to that of a liquid where $D$ is Arrhenius. (c) The same for the ST2 water potential. The liquid-liquid critical point $C$ is located at $P_{c}=246 \mathrm{MPa}$ and $T_{c}=146 \mathrm{~K}$. (d) $D$ as a function of $T$ for $P=100 \mathrm{MPa}$ (path $\alpha$ ). At high temperatures, $D$ behaves like that of a non-Arrhenius liquid and can be fit by $D \sim\left(T-T_{M C T}\right)^{\gamma}$ (also shown in the inset) where $T_{M C T}=239 \mathrm{~K}$ and $\gamma=1.57$, while at low temperatures the dynamic behavior changes to that of a liquid where $D$ is Arrhenius. (e) Phase diagram for the Jagla potential in the vicinity of the liquid-liquid phase transition. Shown are the liquidliquid critical point $C$ located at $P_{c}=0.24 U_{0} / a^{3}$ and $T_{c}=0.37 U_{0} / k_{B}$ (where $U_{0}$ is the atraction energy and $a$ is molecular diameter of the Jagla model), the line of isobaric specific heat maximum $C_{P}^{\max }$, the line of isothermal compressibility $K_{T}^{\mathrm{max}}$, and spinodal lines. (f) $D$ as a function of $T$ for $P=0.250 U_{0} / a^{3}$ (squares, path $\alpha$ ) and $P=0.225 U_{0} / a^{3}$ (triangles, path $\beta$ ). Along path $\alpha$, one can see a sharp crossover from the high temperature Arrhenius behavior $D \approx \exp (-1.53 / T)$ with lower activation energy to a low temperature Arrhenius behavior $D \approx \exp (-6.3 / T)$ with high activation energy, which is a characteristic of the HDL. Along path $\beta$, there is no sharp changes near the critical point, because the liquid remains in the LDL phase. However, near the glass transition, LDL exhibits a non-Arrhenius behavior characterized by the VFT fit at very low temperature. Adapeted from Ref. [8] 
predicts an Arrhenius behavior of $D$. For both water and the Jagla model, crossing the Widom line is associated with the change in the behavior of the diffusivity. (i) In the case of water, $D$ changes from non-Arrhenius to Arrhenius behavior, while the structural and thermodynamic properties change from those resembling HDL to those resembling LDL, due to the negative slope of the Widom line. (ii) For the Jagla potential, $D$ changes from Arrhenius to non-Arrhenius while the structural and thermodynamic properties change from those resembling LDL to those resembling HDL, due to the positive slope of the Widom line (Figure 3).

Thus these results for bulk water are consistent with the experimental observation in confined water of (i) a dynamic crossover for $P<P_{c}[7,13]$, and (ii) a peak in $C_{P}$ upon cooling water at atmospheric pressure [46], so this work offers a plausible interpretation of the results of Ref. [7] as supporting the existence of a hypothesized liquid-liquid critical point.

\section{Hydrated biomolecules}

Next we report about the hypothesis [47] that the so called "glass transition" observed in hydrated biomolecules [48-62] is related to the dynamic crossover described in the previous section. We use quotations for the expression "glass transition" because both terms "glass" and "transition" are used in the literature with an extended meaning. In reality, they refers to a dynamic change observed in hydrated biomolecules. We report here the study by Kumar et al. [47] on the dynamic and thermodynamic behavior of lysozyme and DNA in hydration TIP5P water, performed by means of the molecular dynamics software package GROMACS [63] for (i) an orthorhombic form of hen egg-white lysozyme [64] and (ii) a Dickerson dodecamer DNA [65] at constant pressure $P=1 \mathrm{~atm}$, several constant temperatures $T$, and constant number of water molecules $N$ (NPT ensemble).

The simulation results for the mean square fluctuations $\left\langle x^{2}\right\rangle$ of both protein and DNA are shown in Figure 4. The mean square fluctuations $\left\langle x^{2}\right\rangle$ of the biomolecules is calculated from the equilibrated configurations, first for each atom over $1 \mathrm{~ns}$, and then averaged over the total number of atoms in the biomolecule. Kumar et al. found that $\left\langle x^{2}\right\rangle$ changes its functional form below $T_{\mathrm{p}} \approx 245 \mathrm{~K}$, for both lysozyme [Figure 4(a)] and DNA [Figure 4(b)].

Kumar et. al., next calculated $C_{P}$ by numerical differentiation of the total enthalpy of the system (protein and water) by fitting the simulation data for enthalpy with a fifth order polynomial, and then taking the derivative with respect to $T$. Figures $5(\mathrm{a})$ and $5(\mathrm{~b})$ display maxima of $C_{P}(T)$ at $T_{\mathrm{W}} \approx$ $250 \pm 10 \mathrm{~K}$ for both biomolecules.

Further, to describe the quantitative changes in structure of hydration water, Kumar et al. calculated the local tetrahedral order parameter $Q$ [66] for hydration water surrounding lysozyme and DNA. Figures $5(\mathrm{c})$ and $5(\mathrm{~d})$ show that the rate of increase of $Q$ has a maximum at $245 \pm 10 \mathrm{~K}$ for lysozyme 

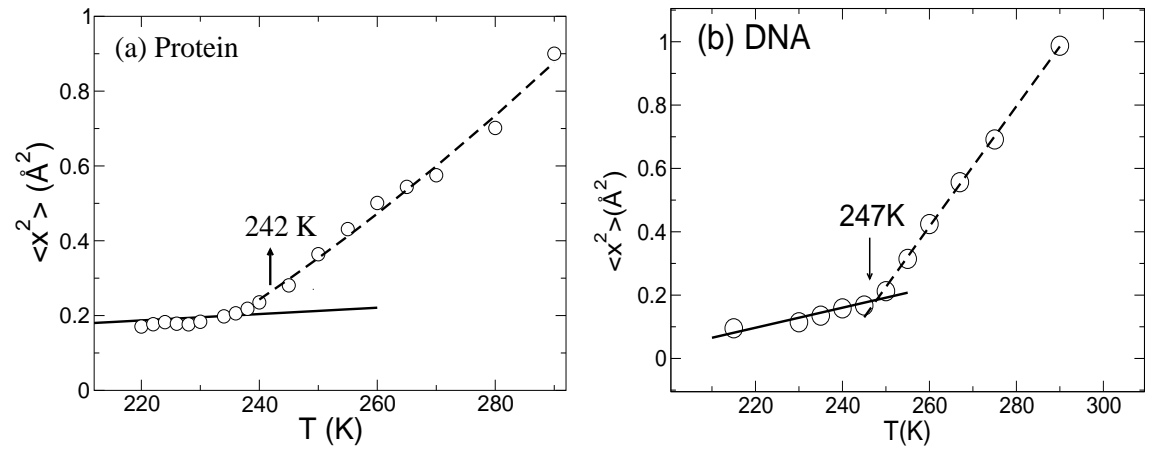

Fig. 4. Mean square fluctuation of (a) lysozyme, and (b) DNA showing that there is a transition around $T_{\mathrm{p}} \approx 242 \pm 10 \mathrm{~K}$ for lysozyme and around $T_{\mathrm{p}} \approx 247 \pm 10 \mathrm{~K}$ for DNA. For very low $T$ one would expect a linear increase of $\left\langle x^{2}\right\rangle$ with $T$, as a consequence of harmonic approximation for the motion of residues. At high $\mathrm{T}$, the motion becomes non-harmonic and is fitted by a polynomial. The dynamic crossover temperature $T_{\mathrm{p}}$ is determined from the crossing of the linear fit for low $\mathrm{T}$ and the polynomial fit for high $\mathrm{T}$. The error bars is determined by changing the number of data points in the two fitting ranges. From Ref. [47].

and DNA hydration water respectively; the same temperatures of the crossover in the behavior of mean square fluctuations.

Upon cooling, the diffusivity of hydration water exhibits a dynamic crossover from non-Arrhenius to Arrhenius behavior at the crossover temperature $T_{\times} \approx 245 \pm 10 \mathrm{~K}$ for lysozyme [Figure $\left.5(\mathrm{e})\right]$ and $T_{\times} \approx 250 \pm 10 \mathrm{~K}$ for DNA [Figure 5(f)]. The coincidence of $T_{\times}$with $T_{\mathrm{p}}$ within the error bars indicates that the behavior of the protein and the DNA is strongly coupled with the behavior of the surrounding solvent, in agreement with recent experiments on hydrated protein and DNA [48] which found the crossover in side-chain fluctuations at $T_{\mathrm{p}} \approx 225 \mathrm{~K}$ [48]. Note that $T_{\times}$is much higher than the glass transition temperature, estimated for TIP5P as $T_{g}=215 \mathrm{~K}$ [67]. Thus this crossover is not likely to be related to the glass transition in water.

The fact that $T_{\mathrm{p}} \approx T_{\times} \approx T_{\mathrm{W}}$ is evidence of the correlation between the changes in protein fluctuations [Figure 4(a)] and the hydration water thermodynamics [Figure 5(a)]. Thus these results are consistent with the possibility that the protein "glass transition" is related to the Widom line. In the next section we will explore with more details the relation between the thermodynamics, structural properties and dynamics in the vicinity of the Widom line. Here we observe that crossing the Widom line corresponds to a continuous but rapid transition of the properties of water from those resembling the properties of a local HDL structure for $T>T_{\mathrm{W}}(P)$ to those resembling the properties of a local LDL structure for $T<T_{\mathrm{W}}(P)[7,8,47]$. A consequence is the expectation that the fluctuations of the protein residues in predominantly LDL-like water (more ordered and more rigid) just below the Widom 

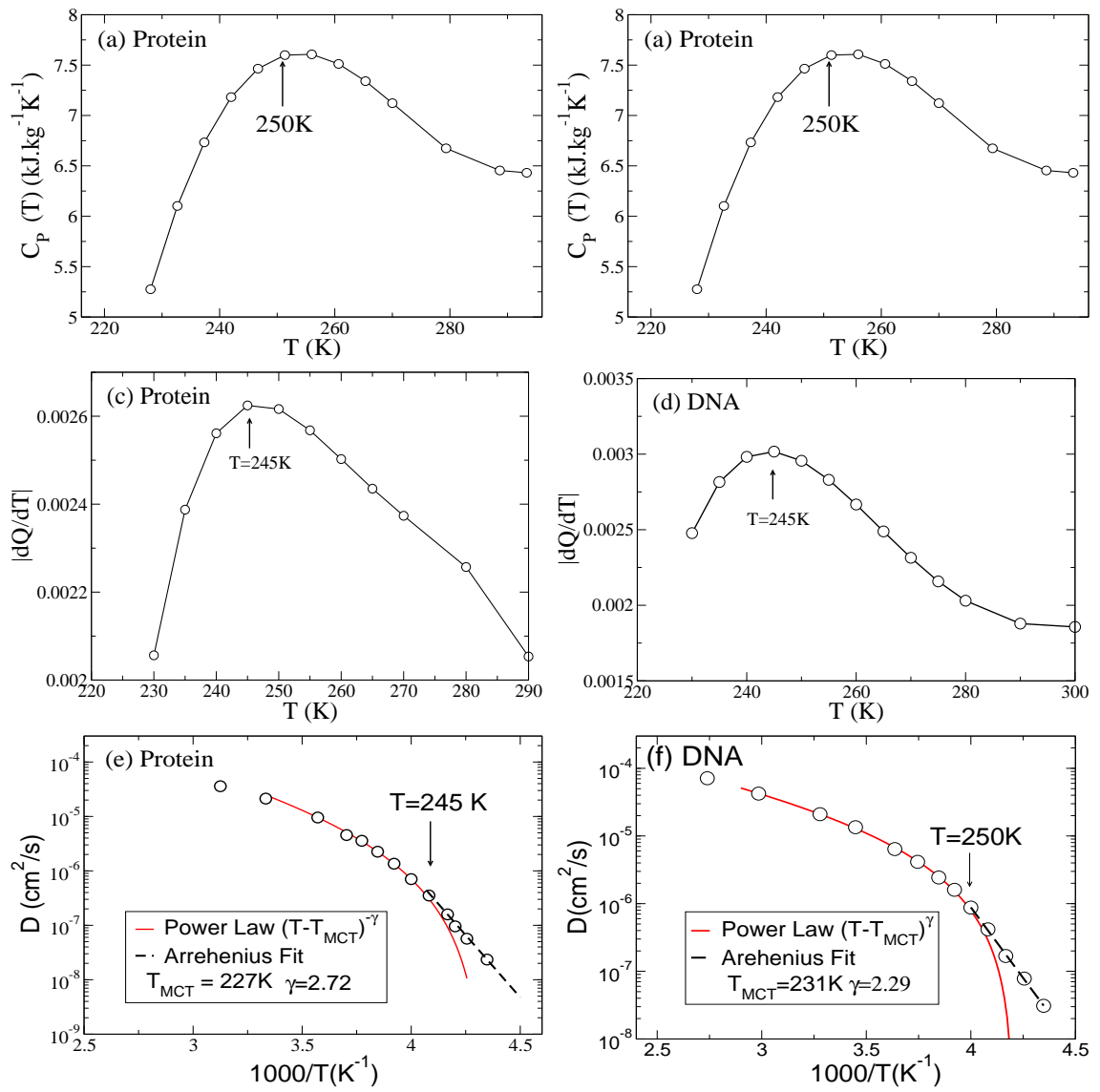

Fig. 5. The specific heat of the combined system (a) lysozyme and water, and (b) DNA and water, display maxima at $250 \pm 10 \mathrm{~K}$ and $250 \pm 12 \mathrm{~K}$ respectively, which are coincident within the error bars with the temperature $T_{\mathrm{p}}$ where the crossover in the behavior of $\left\langle x^{2}\right\rangle$ is observed in Figure 4. Derivative with respect to temperature of the local tetrahedral order parameter $Q$ for (c) lysozyme and (d) DNA hydration water. A maximum in $|d Q / d T|$ at Widom line temperature suggests that the rate of change of local tetrahedrality of hydration water has a maximum at the Widom line. Diffusion constant of hydration water surrounding (e) lysozyme, and (f) DNA shows a dynamic transition from a power law behavior to an Arrhenius behavior at $T_{\times} \approx 245 \pm 10 \mathrm{~K}$ for lysozyme and $T_{\times} \approx 250 \pm 10 \mathrm{~K}$ for DNA, around the same temperatures where the behavior of $\left\langle x^{2}\right\rangle$ has a crossover, and $C_{P}$ and $|d Q / d T|$ have maxima. From Ref. [47]. 
line should be smaller than the fluctuations in predominantly HDL-like water (less ordered and less rigid) just above the Widom line.

The quantitative agreement of the results for both DNA and lysozyme [Figures 4 and 5] suggests that it is indeed the changes in the properties of hydration water that are responsible for the changes in dynamics of the protein and DNA biomolecules. All the results suggest that these changes occur at the Widom line.

\section{Other evidences of changes at the Widom line}

In the previous section we concentrated on reviewing the evidence for changes in dynamic transport properties, such as diffusion constant and relaxation time upon crossing the Widom line. In addition, a series of other phenomena has been observed in computer simulations and experiments around the Widom line:

- A sharp breakdown of Stokes-Einstein relation and a data collapse if $T$ is replaced by $T-T_{W}[68-70]$.

- The appearance of a "boson peak" on cooling below the Widom line [68].

- The occurrence of a new transport mechanism below the Widom line, showing "dynamic heterogeneities" [68-70].

- A dynamic crossover in the relaxation time for $Q$ fluctuations below the Widom line [71].

- Structural changes in $\mathrm{g}(\mathrm{r})$ and its Fourier transform $S(q)$ [72].

- A sharp drop in $T$ derivative of zero-frequency structure factor.

It is possible that all these phenomena that appear on crossing the Widom line are in fact not coincidences, but are related to the changes in local structure occurring when the system goes from the HDL-like side to the LDL-like side of the Widom line. To understand better this possibility we report an investigation [73] of a tractable model, in which the simulations can be complemented with analytic calculations. In Ref. [73], indeed, it has been found an explicit relation between the thermodynamics and the dynamics that allows to calculate the crossover directly from thermodynamics quantities. Since the crossover appears to be related only to the properties of water, the authors focus on the behavior of bulk water to reduce the complexity of the analysis.

\section{Relation between thermodynamics and dynamics}

In Ref. [73], Kumar et al. propose and verify a relation between dynamics and thermodynamics that shows how the crossovers described in previous sections are a direct consequence of the structural change at the temperature of the specific heat maximum $T\left(C_{P}^{\max }\right)$. They adopt a tractable model for water [7477 and express the relevant free energy barrier for the local rearrangement of 
the molecules in terms of the probability $p_{\mathrm{B}}$ of forming bonds. By mean field calculations and Monte Carlo simulations they find that the variation of $p_{\mathrm{B}}$ is the largest at $T\left(C_{P}^{\max }\right)$. If the phase diagram displays a liquid-liquid critical point, $T\left(C_{P}^{\max }\right)$ coincides with $T_{W}(P)$, the Widom line. On approaching the maximum pressure $P_{W}^{\max }$ along $T_{W}(P)$, the crossover changes from sharp, as in water at ambient $P$ [7], to smooth, a result that is explained in terms of the $P$-dependence of the free energy barrier.

We consider a cell model that reproduces the fluid phase diagram of water and other tetrahedral network forming liquids [74-77]. For sake of clarity, we focus on water to explain the motivation of the model. The model is based on the experimental observations that on decreasing $P$ at constant $T$, or on decreasing $T$ at constant $P$, (i) water displays an increasing local tetrahedrality $[78,79]$, (ii) the volume per molecule increases at sufficiently low $P$ or $T$, and (iii) the O-O-O angular correlation increases [80], consistent with simulations $[81,82]$.

The system is divided into cells $i \in[1, \ldots, N]$ on a regular square lattice, each containing a molecule with volume $v \equiv V / N$, where $V \geq N v_{0}$ is the total volume of the system, and $v_{0}$ is the hard-core volume of one molecule. The cell volume $v$ is a continuous variable that gives the mean distance $r \equiv v^{1 / d}$ between molecules in $d$ dimensions. The van der Waals attraction between the molecules is represented by a truncated Lennard-Jones potential with characteristic energy $\epsilon>0$

$$
U(r) \equiv \begin{cases}\infty & \text { for } r \leq R_{0} \\ \epsilon\left[\left(\frac{R_{0}}{r}\right)^{12}-\left(\frac{R_{0}}{r}\right)^{6}\right] & \text { for } r>R_{0},\end{cases}
$$

where $R_{0} \equiv v_{0}^{1 / d}$ is the hard-core distance [74-77].

Each molecule $i$ has four bond indices $\sigma_{i j} \in[1, \ldots, q]$, corresponding to the nearest-neighbor cells $j$. When two nearest-neighbor molecules have the facing $\sigma_{i j}$ and $\sigma_{j i}$ in the same relative orientation, they decrease the energy by a constant $J$, with $0<J<\epsilon$, and form a (non-bifurcated) hydrogen bond. The choice $J<\epsilon$ guarantees that bonds are formed only in the liquid phase. The bond interaction is accounted for by a term in the Hamiltonian

$$
\mathcal{H}_{\mathrm{B}} \equiv-J \sum_{\langle i, j\rangle} \delta_{\sigma_{i j} \sigma_{j i}}
$$

where the sum is over nearest-neighbor cells, and $\delta_{a, b}=1$ if $a=b$ and $\delta_{a, b}=0$ otherwise. For water at high $P$ and $T$ a more dense, collapsed and distorted, local structure with bifurcated hydrogen bonds is consistent with the experiments. Bifurcated hydrogen bonds decrease the strength of the network and favor the hydrogen bond breaking and re-formation. The model simplifies the situation by assuming that (a) only non-bifurcated, i.e. normal, hydrogen bond decrease the energy of the system and (b) the local density changes as function of the number of normal hydrogen bonds, consistent with the observation [80] that at low $P$ and $T$ there is a better separation between the first 
neighbors and the second neighbors, favoring normal hydrogen bonds and the tetrahedral order.

The density decrease for $T<T_{\mathrm{MD}}(P)$, the temperature of maximum density, is represented by an average increase of the molar volume due to a more structured network. If the total volume increases by an amount $v_{\mathrm{B}}>0$ for each bond formed [4, 74-77], then the molar volume is

$$
v=v^{\prime}+2 p_{\mathrm{B}} v_{\mathrm{B}}
$$

where $v^{\prime}$ is the molar volume without taking into account the bond and $p_{\mathrm{B}}$ is the probability of forming a bond. The increase of the angular molecular correlation is modeled by introducing an intramolecular (IM) interaction of energy $0<J_{\sigma}<J$,

$$
\mathcal{H}_{\mathrm{IM}} \equiv-J_{\sigma} \sum_{i} \sum_{(k, \ell)_{i}} \delta_{\sigma_{i k} \sigma_{i \ell}},
$$

where $\sum_{(k, \ell)_{i}}$ denotes the sum over the bond indices of the molecule $i$.

The total energy of the system is the sum of Eqs. (3), (4) and (6). They perform mean field calculations and Monte Carlo simulations in the NPT ensemble [74-77] for a system with $J / \epsilon=0.5, J_{\sigma} / \epsilon=0.05, v_{\mathrm{B}} / v_{0}=0.5$, $q=6$. The study of two square lattices with 900 and 3600 cells shows no appreciable size effects. Below the $T_{\mathrm{MD}}$ line, in the supercooled region, the model displays a first-order phase transition between a LDL at low $P$ and $T$ and a HDL at high $P$ and $T$ along a line terminating in the liquid-liquid critical point $C^{\prime}$ [74-77] [Fig. 6(a)]. For $J_{\sigma} \rightarrow 0, C^{\prime}$ moves toward $T=0$ [83] suggesting that the singularity free scenario is a limiting case of the liquidliquid critical point scenario [83]. For $J_{\sigma}>0, P_{W}^{\max }$ coincides with $P_{C^{\prime}}$, while for $J_{\sigma}=0$ it is a point on the $T=0$ axis [83].

Kumar et al. find that different response functions such as $C_{P}, \alpha_{P}$ [Figure $6(\mathrm{~b})$ and $6(\mathrm{c})$ ] show maxima and these maxima increase and seem to diverge as the critical pressure is approached, consistent with the picture of Widom line that we discussed for other water models in the sections above. Moreover, the temperature derivative of the probability of forming hydrogen bonds $d p_{B} / d T$ displays a maximum in the same region where the other thermodynamic response functions have maxima, suggesting that the fluctuations in the $p_{B}$ is maximum at the Widom line temperature $T_{W}(P)$ [Figure $6(\mathrm{~d})$ ].

To further test if this model system also displays a dynamic crossover as found in the other models of water, the total spin relaxation time of the system as a function of $T$ for different pressures is studied. The results show that the liquid is more non-Arrhenius for increasing $P$ [Figure 7(a)]. For $P=0$ [Fig. 7(b)] by decreasing $T$ there is a crossover from Arrhenius to the VogelFulcher-Tamman (VFT) function

$$
\tau^{\mathrm{VFT}}=\tau_{0}^{\mathrm{VFT}} \exp \left[\frac{T_{1}}{T-T_{0}}\right],
$$



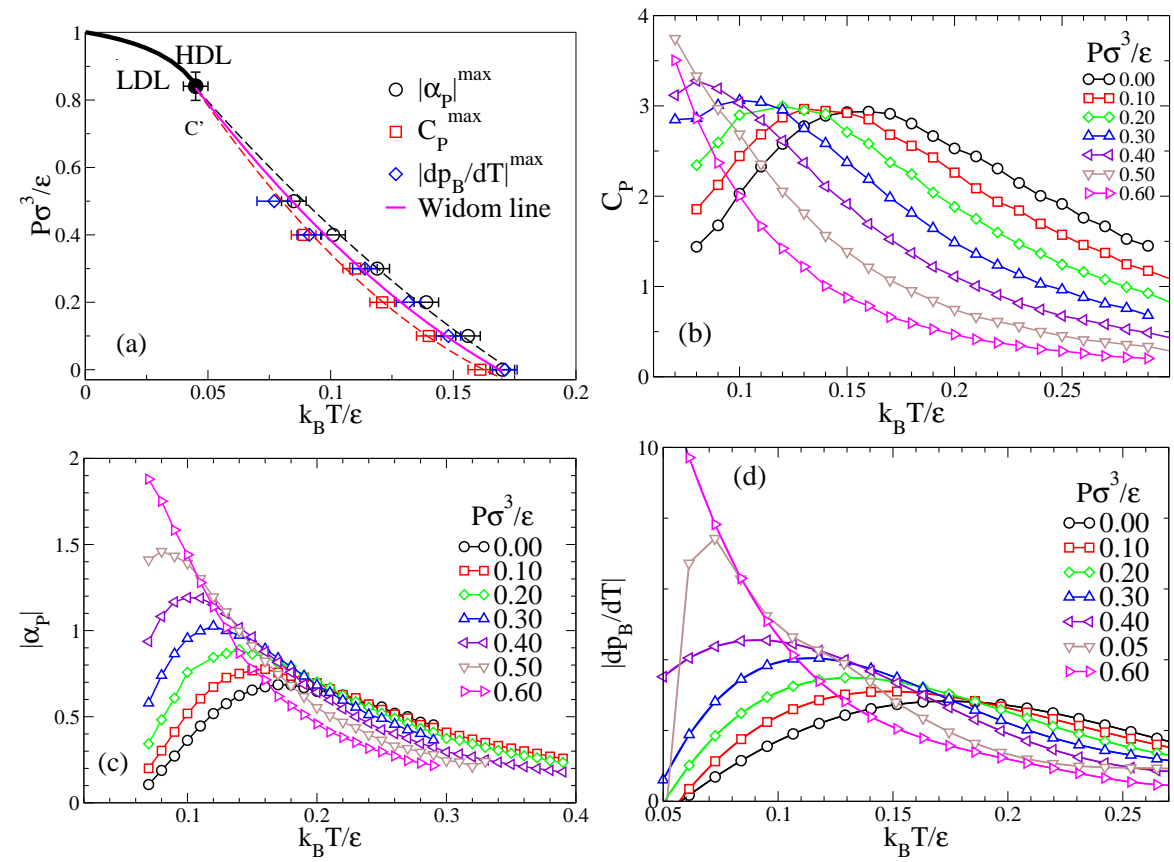

Fig. 6. (a) Phase diagram below $T_{\mathrm{MD}}$ line shows that $\left|d p_{\mathrm{B}} / d T\right|^{\max }(\diamond)$ coincides with the Widom line $T_{W}(P)$ (solid line) within error bars: $C^{\prime}$ is the HDL-LDL critical point, end of first-order phase transition line (thick line) [74-77]; symbols are maxima for $N=3600$ of $\left|\alpha_{P}\right|^{\max }(\bigcirc), C_{P}^{\max }(\square)$, and $\left|d p_{\mathrm{B}} / d T\right|^{\max }(\diamond)$; upper and lower dashed line are quadratic fits of $\left|\alpha_{P}\right|^{\max }$ and $C_{P}^{\max }$, respectively, consistent with $C^{\prime} ;\left|\alpha_{P}\right|^{\max }$ and $C_{P}^{\max }$ are consistent within error bars. Maxima are estimated from panels (b), (c) and (d), where each quantity is shown as functions of $T$ for different $P<P_{C^{\prime}}$. In (d) $\left|d p_{\mathrm{B}} / d T\right|^{\max }$ is the numerical derivative of $p_{\mathrm{B}}$ from simulations. From Ref. [73].

where $\tau_{0}^{\mathrm{VFT}}, T_{1}$ and $T_{0}$ are all fitting parameters. At lower temperatures there is another crossover from VFT back to Arrhenius. The Arrhenius activation energy at low $T$ is higher than that at high $T$, consistent with experiments at ambient $P$ for both bulk water [30, 84] and confined water [7, 39].

As the pressure is increased towards the critical pressure $P_{C}$ of the system, this dynamic crossover in $\tau$ at low $T$ gets smoother [Figure $7(\mathrm{c})$ ] suggesting that the dynamics of water will be more non-Arrhenius as the critical point is approached.

For all $P$ the crossover occurs at $T_{W}(P)$ within the error bars [Fig. $7(\mathrm{c})$ ], confirming the idea proposed on the base of simulations of detailed models for water reported in the previous sections $[8,47]$. We observe that the low- $T$ behavior is characterized by an activation energy - the slope in Fig. 7(c) - that decreases for increasing $P$, as in experiments for confined water [7], and that the time needed to reach the maximum correlation length is almost indepen- 

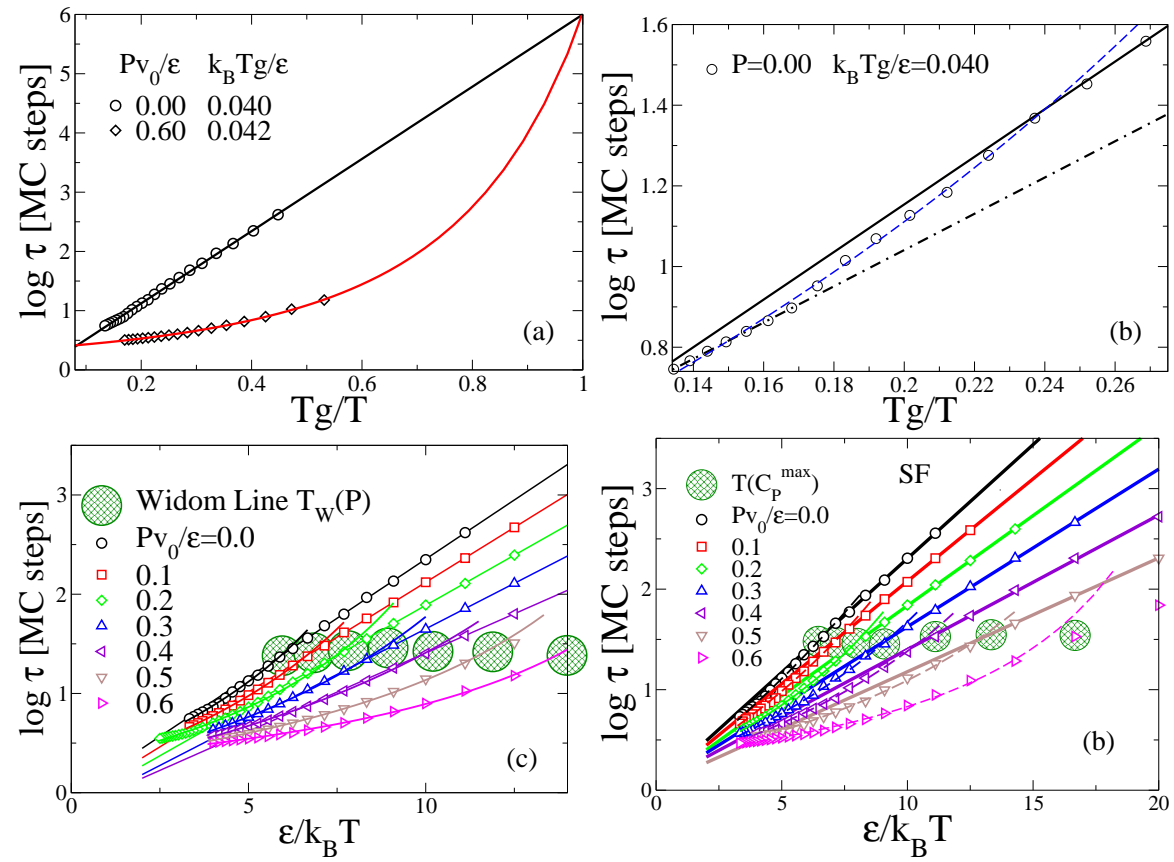

Fig. 7. (a) Angell plot of logarithm of relaxation time $\tau$ as a function of $T_{g} / T$ shows a large difference between $P v_{0} / \epsilon=0$ and $0.6 ; T_{g}$ is defined in such a way that $\tau\left(T_{g}\right)=10^{6} \mathrm{MC}$ steps $\sim 10^{7} \mathrm{ps}[26]$; for $P v_{0} / \epsilon=0.6(\diamond)$ the $\tau$ can be described as a VFT function Eq. (7) (curved line), while for $P=0(\bigcirc)$ as an Arrhenius function (straight line). Note that the data for $P v_{0} / \epsilon=0.6$ extends only down to temperatures where the system has non-Arrhenius dynamics. This suggests that the system becomes increasingly non-Arrhenius as the pressure is increased. (b) A detailed observation at $P=0$ shows three regimes: Arrhenius at low $T_{g} / T$ with low activation energy (slope of dash-dot line), non-Arrhenius (VFT, dash line) at intermediate $T$, and again Arrhenius at high $T_{g} / T$ with higher activation energy (slope of solid line) as in experiments on water. (c) At $0 \leq P v_{0} / \epsilon \leq 0.4$ the Arrhenius crossover occurs at $T$ consistent with $T_{W}(P)$ (large $\boxplus$ ); the error on $T_{W}(P)$ is approximately equal to symbol size; thick and thin lines represent VFT and Arrhenius fits. (d) Dynamic crossover for the singularity free (SF) scenario, with crossover temperature at $T\left(C_{P}^{\max }\right)$. Solid and dashed lines represent Arrhenius and non-Arrhenius fits, respectively. Notice that the dynamic crossover occurs at approximately the same value of $\tau$ for all seven values of pressure studied, as in panel (c). 
dent of the position along $T_{W}(P)$, being $\log \tau\left(T_{W}\right) \simeq 1.5 \mathrm{MC}$ steps $\simeq 15 \mathrm{ps}$ [26] for any $P$. For completeness we study the system also in the case of singularity free scenario, corresponding to $J_{\sigma}=0$. For $J_{\sigma}=0$ the crossover is at $T\left(C_{P}^{\max }\right)$, the temperature of $C_{P}^{\max }[$ Fig. $7(\mathrm{~d})]$. Kumar et al. next calculate the Arrhenius activation energy $E_{\mathrm{A}}(P)$ from the low- $T$ slope of $\log \tau$ vs. $1 / T$ [Fig. 8(a)]. They extrapolate the temperature $T_{\mathrm{A}}(P)$ at which $\tau$ reaches a fixed macroscopic time $\tau_{\mathrm{A}} \geq \tau_{\mathrm{C}}$. We choose $\tau_{\mathrm{A}}=10^{14} \mathrm{MC}$ steps $>100 \mathrm{sec}[26]$ [Fig. 8(b)]. We find that $E_{\mathrm{A}}(P)$ and $T_{\mathrm{A}}(P)$ decrease upon increasing $P$ in both scenarios, providing no distinction between the two interpretations. Instead, we find a dramatic difference in the $P$ dependence of the quantity $E_{\mathrm{A}} /\left(k_{B} T_{\mathrm{A}}\right)$ in the two scenarios, increasing for the liquid-liquid critical point scenario and approximately constant for the singularity free scenario [Fig. 8(c)].
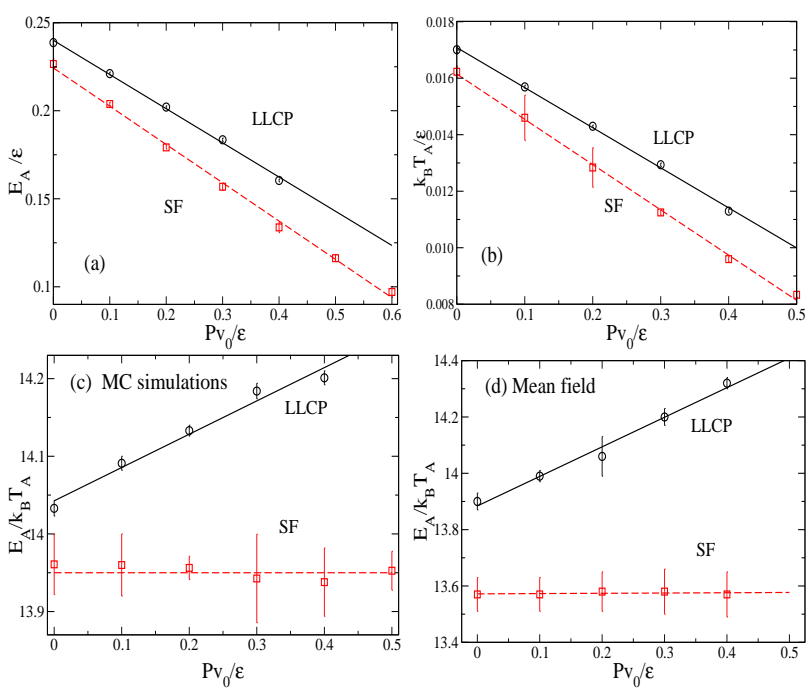

Fig. 8. Effect of pressure on the activation energy $E_{\mathrm{A}}$. (a) Demonstration that $E_{\mathrm{A}}$ decreases linearly for increasing $P$ for both the liquid-liquid critical point (LLCP) and the singularity free (SF) scenarios. The lines are linear fits to the simulation results (symbols). (b) $T_{\mathrm{A}}$, defined such that $\tau\left(T_{\mathrm{A}}\right)=10^{14} \mathrm{MC}$ steps $>100 \mathrm{sec}$ [26], decreases linearly with $P$ for both scenarios. (c) $P$ dependence of the quantity $E_{\mathrm{A}} /\left(k_{B} T_{\mathrm{A}}\right)$ is different in the two scenarios. In the LLCP scenario, $E_{\mathrm{A}} /\left(k_{B} T_{\mathrm{A}}\right)$ increases with increasing $P$, and it is approximately constant in the SF scenario. The lines are guides to the eyes. (d) Demonstration that the same behavior is found using the mean field approximation. In all the panels, where not shown, the error bars are smaller than the symbol sizes.

To better understand their findings, Kumar et al. [73] develope an expression for $\tau$ in terms of thermodynamic quantities, which allows to explicitly calculate $E_{\mathrm{A}} /\left(k_{B} T_{\mathrm{A}}\right)$ for both scenarios. For any activated process, in which the relaxation from an initial state to a final state passes through an excited 
transition state, $\ln \left(\tau / \tau_{0}\right)=\Delta(U+P V-T S) /\left(k_{B} T\right)$, where $\Delta(U+P V-T S)$ is the difference in free energy between the transition state and the initial state. Consistent with results from simulations and experiments [85, 86], Kumar et al. propose that at low $T$ the mechanism to relax from a less structured state (lower tetrahedral order) to a more structured state (higher tetrahedral order) corresponds to the breaking of a bond and the simultaneous molecular reorientation for the formation of a new bond. The transition state is represented by the molecule with a broken bond and more tetrahedral IM order. Hence,

$$
\Delta(U+P V-T S)=J p_{\mathrm{B}}-J_{\sigma} p_{\mathrm{IM}}-P v_{\mathrm{B}}-T \Delta S,
$$

where $p_{\mathrm{B}}$ and $p_{\mathrm{IM}}$, the probability of a satisfied IM interaction, can be directly calculated. To estimate $\Delta S$, the increase of entropy due to the breaking of a bond, they use the mean field expression $\Delta S=k_{B}\left[\ln \left(2 N p_{\mathrm{B}}\right)-\ln (1+2 N(1-\right.$ $\left.\left.\left.p_{\mathrm{B}}\right)\right)\right] \bar{p}_{\mathrm{B}}$, where $\bar{p}_{\mathrm{B}}$ is the average value of $p_{\mathrm{B}}$ above and below $T_{W}(P)$.

They next test that the expression of $\ln \left(\tau / \tau_{0}\right)$, in terms of $\Delta S$ and Eq.(8),

$$
\ln \frac{\tau}{\tau_{0}}=\frac{J p_{\mathrm{B}}-J_{\sigma} p_{\mathrm{IM}}-P v_{\mathrm{B}}}{k_{B} T}-\bar{p}_{\mathrm{B}} \ln \frac{2 N p_{\mathrm{B}}}{1+2 N\left(1-p_{\mathrm{B}}\right)}
$$

describes the simulations well, with minor corrections at high $T$. Here $\tau_{0} \equiv$ $\tau_{0}(P)$ is a free fitting parameter equal to the relaxation time for $T \rightarrow \infty$. From Eq.(9) they find that the ratio $E_{\mathrm{A}} /\left(k_{B} T_{\mathrm{A}}\right)$ calculated at low $T$ increases with $P$ for $J_{\sigma} / \epsilon=0.05$, while it is constant for $J_{\sigma}=0$, as from their simulations [Fig. 8(d)].

\section{Conclusions}

Recent experiments at very low temperatures, below the crystal homogeneous nucleation temperature of bulk water, have revealed the relation between the dynamics of water and that of proteins or DNA [47, 48]. The experiments on hydration water, as well as confined water [7, 39, 40, 87], and the corresponding simulations $[8,47]$, find a change in the water relaxation dynamics that suggests a crossover from a non-Arrhenius dynamics at higher $T$ to Arrhenius dynamics at very low $T$ [8], as predicted for bulk water [30].

The simulations show that the dynamics becomes Arrhenius when the liquid is cooled isobarically below the temperature of maximum specific heat $T\left(C_{P}^{\max }\right)$ and that water is more tetrahedral for $T<T\left(C_{P}^{\max }\right)$ [47]. In one possible interpretation of the anomalous properties of water, $T\left(C_{P}^{\max }\right)$ coincides with the Widom line emanating from a hypothesized liquid-liquid critical point $C^{\prime}[6]$, the terminus of a liquid-liquid phase transition line that separates a low-density liquid and a high-density liquid.

Based on the study of the probability $p_{\mathrm{B}}$ of forming a Hydrogen bond, we have seen that it is possible to show [73] how the crossover at $T\left(C_{P}^{\max }\right)$ is a consequence of a local relaxation process associated with breaking a bond 
and reorienting the molecule. The investigation reported here allow to find the relation between dynamics and thermodynamics in terms of the relevant activation barrier [73]. The crossover at $T\left(C_{P}^{\max }\right)$ is regulated by Eq.(9), as a consequence of a local breaking of a bond and simultaneous molecular reorientation to increase the structural order. This is consistent with what is observed in SPC/E water [85] and with recent experimental conclusions [9]. In Ref. [73] it has been found the expression for the relevant free energy barrier [Eq.(8)] and how it depends on pressure. The barrier rapidly increases with $1 / T$ above $T\left(C_{P}^{\max }\right)$, while has only a weak dependence on $T$ below $T\left(C_{P}^{\max }\right)$, giving rise to the crossover.

In conclusion, the investigations reported here show that the dynamics of water at low temperature is a consequence of the structural changes of the network formed by the hydrogen bonds. Our results suggest that the change in the dynamic behavior of water around the line of maxima of specific heat could be the cause of the so called "glass transition" in hydrated proteins or DNA. Hence the dynamics of biomolecules turns out to be dominated by the changes in the hydration water.

\section{Acknowledgments}

We thank C. A. Angell, W. Kob, L. Liu, F. Mallamace, P. H. Poole, S. Sastry, and NSF for financial support. G. F. also thanks the Spanish Ministerio de Educación y Ciencia (Programa Ramón y Cajal and Grant No. FIS200403454).

\section{References}

[1] F. Franks: Biochemistry and Biophysics at Low Temperatures (Cambridge University Press, Cambridge 1985)

[2] C. A. Angell: Ann. Rev. Phys. Chem. 55, 559 (2004)

[3] H. E. Stanley and J. Teixeira: J. Chem. Phys. 73, 3404 (1980)

[4] S. Sastry, P. G. Debenedetti, F. Sciortino, and H. E. Stanley: Phys. Rev. E 53, $6144(1996)$

[5] P. H. Poole, F. Sciortino, U. Essmann, and H. E. Stanley: Nature (London) 360, 324 (1992)

[6] O. Mishima and H. E. Stanley: Nature (London) 392, 164 (1998)

[7] L. Liu, S.-H. Chen, A. Faraone, C.-W. Yen, and C.-Y. Mou: Phys. Rev. Lett. 95, $117802(2005)$

[8] L. Xu, P. Kumar, S. V. Buldyrev, S.-H. Chen, P. Poole, F. Sciortino, and H. E. Stanley: Proc. Natl. Acad. Sci. U.S.A. 102, 16558 (2005)

[9] J. Swenson: Phys. Rev. Lett. 97, 189801 (2006)

[10] S. Cerveny, J. Colmenero, and A. Alegria: Phys. Rev. Lett. 97, 189802 (2006) 
[11] S.-H. Chen, L. Liu, and A. Faraone: Phys. Rev. Lett. 97, 189803 (2006)

[12] P. G. Debenedetti and H. E. Stanley: Physics Today 56, 40 (2003)

[13] A. Faraone, L. Liu, C. Y. Mou, C. W. Yen, and S. H. Chen: J. Chem. Phys. 121, 10843 (2004)

[14] G. Franzese, G. Malescio, A. Skibinsky, S. V. Buldyrev, and H. E. Stanley: Nature (London) 409, 692 (2001)

[15] G. Franzese: Differences between discontinuous and continuous soft-core attractive potentials: the appearance of density anomaly (2007)

[16] A. B. de Oliveira, G. Franzese, P. A. Netz, and M. C. Barbosa: Water-like hierarchy of anomalies in a continuous spherical shouldered potential. J. Chem. Phys. (in press) (2007), URL http://arxiv.org/abs/0706.2838

[17] M. A. Anisimov, J. V. Sengers, and J. M. H. Levelt Sengers: In: Aqueous System at Elevated Temperatures and Pressures: Physical Chemistry in Water, Steam and Hydrothermal Solutions, ed by D. A. Palmer, R. Fernandez-Prini, and A. H. Harvey (Elsevier, Amsterdam 2004) pp 29 71

[18] J. M. H. Levelt: Measurements of the compressibility of argon in the gaseous and liquid phase. Ph.D. thesis, University of Amsterdam, Van Gorkum and Co., Assen (1958)

[19] A. Michels, J. M. H. Levelt, and G. Wolkers: Physica 24, 769 (1958)

[20] A. Michels, J. M. H. Levelt, and W. de Graaff: Physica 24, 659 (1958)

[21] L. Xu, S. Buldyrev, C. A. Angell, and H. E. Stanley: Phys. Rev. E 74, 031108 (2006)

[22] P. Debenedetti: J. Phys.: Condens. Matter 15, R1669 (2003)

[23] F. W. Starr, C. A. Angell, and H. E. Stanley: Physica A 323, 51 (2003)

[24] C. A. Angell: J. Phys. Chem. 97, 6339 (1993)

[25] P. Kumar, S. V. Buldyrev, F. W. Starr, N. Giovambattista, and H. E. Stanley: Phys. Rev. E 72, 051503 (2005)

[26] P. Kumar, G. Franzese, S. V. Buldyrev, and H. E. Stanley: Phys. Rev. E 73, 041505 (2006)

[27] J. Horbach and W. Kob: Phys. Rev. B 60, 3169 (1999)

[28] E. W. Lang and H.-D. Lüdemann: Angew. Chem. Intl. Ed. Engl. 21, 315 (2004)

[29] F. X. Prielmeier, E. W. Lang, R. J. Speedy, and H.-D. Lüdemann: Phys. Rev. Lett. 59, 1128 (1987)

[30] K. Ito, C. T. Moynihan, and C. A. Angell: Nature (London) 398, 492 (1999)

[31] E. A. Jagla: J. Chem. Phys. 111, 8980 (1999)

[32] E. A. Jagla: J. Phys.: Condens. Matter 11, 10251 (1999)

[33] E. A. Jagla: Phys. Rev. E 63, 061509 (2001)

[34] H. Tanaka: J. Phys. Condens. Matter 15, L703 (2003)

[35] G. Adam and G. H. Gibbs: J. Chem. Phys. 43, 139 (1965)

[36] P. H. Poole, I. Saika-Voivod, and F. Sciortino: J. Phys.: Condens. Matter 17, L431 (2005)

[37] S. Sastry and C. A. Angell: Nature Mater. 2, 739 (2003) 
[38] I. Saika-Voivod, P. H. Poole, and F. Sciortino: Nature (London) 412, 514 (2001)

[39] R. Bergman and J. Swenson: Nature (London) 403, 283 (2000)

[40] F. Mallamace, M. Broccio, C. Corsaro, A. Faraone, U. Wanderlingh, L. Liu, C. Y. Mou, and S. H. Chen: J. Chem. Phys. 124, 161102 (2006)

[41] D. C. Rapaport: The Art of Molecular Dynamics Simulation (Cambridge University Press, Cambridge 1995)

[42] M. W. Mahoney and W. L. Jorgensen: J. Chem. Phys. 112, 8910 (2000)

[43] F. H. Stillinger and A. Rahman: J. Chem. Phys. 57, 1281 (1972)

[44] P. Kumar, S. V. Buldyrev, F. Sciortino, E. Zaccarelli, and H. E. Stanley: Phys. Rev. E 72, 021501 (2005)

[45] L. M. Xu, I. Ehrenberg, S. V. Buldyrev, and H. E. Stanley: J. Phys.: Condens. Matter 18, S2239 (2006)

[46] S. Maruyama, K. Wakabayashi, and M. Oguni: In: Slow Dynamics in Complex Systems: Third International Symposium on Slow Dynamics in Complex Systems, AIP Conf. Proc. No. 708, ed by M. Tokuyama and I. Oppenheim (AIP, New York 2004) pp 675-676

[47] P. Kumar, Z. Yan, L. Xu, M. G. Mazza, S. V. Buldyrev, S.-H. Chen, S. Sastry, and H. E. Stanley: Phys. Rev. Lett. 97, 177802 (2006)

[48] S. H. Chen, L. Liu, E. Fratini, P. Baglioni, A. Faraone, and E. Mamontov: Proc. Natl. Acad. Sci. U.S.A. 103, 9012 (2006)

[49] J.-M. Zanotti, M.-C. Bellissent-Funel, and J. Parello: Biophys. J. 76, 2390 (1999)

[50] D. Ringe and G. A. Petsko: Biophys. Chem. 105, 667 (2003)

[51] Wang JM, Cieplak P, and Kollman PA: J. Comp. Chem. 21, 1049 (2000)

[52] E. J. Sorin and V. S. Pande: Biophys. J. 88, 2472 (2005)

[53] B. F. Rasmussen, A. M. Stock, D. Ringe, and G. A. Petsko: Nature (London) 357, 423 (1992)

[54] D. Vitkup, D. Ringe, G. A. Petsko, and M. Karplus: Nat. Struct. Biol. 7, $34(2000)$

[55] A. P. Sokolov, H. Grimm, and R. Kahn: J. Chem. Phys. 110, 7053 (1999)

[56] W. Doster, S. Cusack, and W. Petry: Nature (London) 337, 754 (1989)

[57] J. Norberg and L. Nilsson: Proc. Natl. Acad. Sci. U.S.A. 93, 10173 (1996)

[58] M. Tarek and D. J. Tobias: Phys. Rev. Lett. 88, 138101 (2002)

[59] M. Tarek and D. J. Tobias: Biophys. J. 79, 3244 (2000)

[60] H. Hartmann, F. Parak, W. Steigemann, G. A. Petsko, D. R. Ponzi, and H. Frauenfelder: Proc. Natl. Acad. Sci. U.S.A. 79, 4967 (1982)

[61] A. L. Tournier, J. Xu, and J. C. Smith: Biophys. J. 85, 1871 (2003)

[62] A. L. Lee and A. J. Wand: Nature (London) 411, 501 (2001)

[63] E. Lindahl, B. Hess, and D. van der Spoel: J. Mol. Mod. 7, 306 (2001)

[64] P. J. Artymiuk, C. C. F. Blake, D. W. Rice, and K. S. Wilson: Acta Crystallogr. B 38, 778 (1982)

[65] H. R. Drew, R. M. Wing, T. Takano, C. Broka, S. Tanaka, K. Itakura, and R. E. Dickerson: Proc. Natl. Acad. Sci. U.S.A. 78, 2179 (1981)

[66] J. R. Errington and P. D. Debenedetti: Nature (London) 409, 318 (2001) 
[67] I. Brovchenko, A. Geiger, and A. Oleinikova: J. Chem. Phys. 123, 044515 (2005)

[68] S.-H. Chen, F. Mallamace, C.-Y. Mou, M. Broccio, C. Corsaro, A. Faraone, and L. Liu: Proc. Natl. Acad. Sci. U.S.A. 103, 12974 (2006)

[69] P. Kumar: Proc. Natl. Acad. Sci. U.S.A. 103, 12955 (2006)

[70] P. Kumar, S. V. Buldyrev, S. R. Becker, P. H. Poole, F. W. Starr, and H. E. Stanley: Proc. Natl. Acad. Sci. U.S.A. 104, 9575 (2007)

[71] P. Kumar, S. V. Buldyrev, and H. E. Stanley: (2007), (preprint)

[72] P. Kumar, S. V. Buldyrev, and H. E. Stanley: In: Soft Matter under Extreme Pressures: Fundamentals and Emerging Technologies, ed by S. J. Rzoska and V. Mazur, Proc. NATO ARW, Odessa, Oct. 2005 (Springer, Berlin Heidelberg New York 2006)

[73] P. Kumar, G. Franzese, and H. E. Stanley: Predictions of dynamic behavior under pressure for two scenarios to explain water anomalies (2007), URL http://arxiv.org/abs/cond-mat/0702108

[74] G. Franzese and H. E. Stanley: J. Phys.: Condens. Matter 14, 2201 (2002)

[75] G. Franzese and H. E. Stanley: Physica A 314, 508 (2002)

[76] G. Franzese, M. I. Marques, and H. E. Stanley: Phys. Rev. E 67, 011103 (2003)

[77] G. Franzese and H. E. Stanley: J. Phys.: Condens. Matter 19, 205126 (2007)

[78] G. Darrigo, G. Maisano, F. Mallamace, P. Migliardo, and F. Wanderlingh: J. Chem. Phys. 75, 4264 (1981)

[79] Angell, C.A. and Rodgers, V.: J. Chem. Phys. 80, 6245 (1984)

[80] A. K. Soper and M. A. Ricci: Phys. Rev. Lett. 84, 2881 (2000), and references cited therein

[81] E. Schwegler, G. Galli, and F. Gygi: Phys. Rev. Lett. 84, 2429 (2000)

[82] P. Raiteri, A. Laio, and M. Parrinello: Phys. Rev. Lett. 93, 087801 (2004), and references cited therein

[83] K. Stokely, G. Franzese, and H. E. Stanley: (2007), (preprint)

[84] I. Kohl, L. Bachmann, A. Hallbrucker, E. Mayer, and T. Loerting: Phys. Chem. Chem. Phys. 7, 3210 (2005)

[85] Laage, D. and Hynes, J. T.: Science 311, 832 (2006)

[86] Tokmakoff, A.: Science 317, 54 (2007)

[87] M. A. Ricci, F. Bruni, P. Gallo, M. Rovere, and A. K. Soper: J. Phys.: Condens. Mat. 12, A345 (2000) 